\title{
Simulation Study for Interface Crack Propagation in Layered Salt Rock Subjected to Compress and Shear Load
}

\author{
Qingnan $\mathrm{Fu}^{\mathrm{a}}$, Yanxia $\mathrm{Wu}^{\mathrm{b}}$ \\ College of Mechanics, Taiyuan University of Technology, Taiyuan 030024, China; \\ afqn614@163.com, bwu_yanxia@163.com
}

\begin{abstract}
Keywords: Layered rock salt, compress and shear load, interface crack, extended finite element method (XFEM).
\end{abstract}

\begin{abstract}
In order to study the effects of interlayer strength, interface strength and interface dip angle on the propagation of interface crack in layered salt rock subjected to compress and shear load, traction-separation cohesive behavior is introduced within the framework of extended finite element method (XFEM) to simulate the crack propagation. The Abaqus software is used to simulate the process of crack propagation in layered salt rock with tilted interface and under uniaxial compression. The stimulation results indicate that new cracks which deviate from original crack will appear under compress and shear load. The influences of interlayer type and interface strength on interface crack are mainly in terms of crack length. Meanwhile, the length and initiation angle of crack are both influenced by interface dip angle. The crack length in layered salt rock containing weak interlayer is larger than that of salt rock containing hard interlayer. The increase of interface strength will lead to the increased crack length, while the increased inclination of interface will shorten the length and crack initiation angle.
\end{abstract}

\section{Introduction}

Due to its great physical and mechanical features such as densification, self-healing performance and sound plastic deformation capacity, salt rock is internationally regarded as the ideal medium for storing oil and natural gas, handling the radioactive nuclear waste and storing compressed air energy [1]. Scientists and engineers have spent more than 50 years in studying the usage of salt caverns, and the study on natural gas storage in salt rock in China was also launched over a decade. Different from the massive salt domes in North America, salt rock deposits in China always contain interlayers. According to previous studies, in layered salt rock, the mechanical property of the interface between salt rock and interlayer has great influence on the strength and damage of layered salt rock [2]. The misfit mechanical properties of salt rock and interlayer always leads to stress concentration and crack initiation at and near the interface. The present studies of interface cracks mainly focus on the composite and less stimulations about propagation process of interface cracks are launched [3]. Shear-compression fracture is important fracture forms of rock-like materials which include layered salt rock. There are many studies related to shear-compression fracture on rock-like materials [4], while few studies are launched on the shear-compression fracture of salt rock. In terms of the crack propagation of layered salt rock, $\mathrm{Li}$ et al. [5] conducted an experimental study on the surface crack propagation and dispersion in layered salt rock under uniaxial compression. Peng et al. [6] conducted real-time observation on the crack extension of salt rock samples containing interlayers in the three-point bending test with the usage of SEM, and explained the test result from the perspective of energy dissipation.

In order to further study the propagation features of interface crack in layered salt rock, traction-separation cohesive behavior as well as extended finite element method (XFEM) is adopted in this paper to stimulate the overall process of interface crack propagation in layered salt rock with the usage of Abaqus. Influences of interlayer strength, interface strength and interface dip angle on interface crack propagation in layered salt rock under shear-compression load are discussed. 


\section{Calculation Model and Parameters}

As showed in Figure 1, the computational model is a rectangular flat with the height $2 \mathrm{H}=400 \mathrm{~mm}$ and width $\mathrm{W}=100 \mathrm{~mm}$. The upper part is salt rock and the lower part is interlayer (including weak interlayer and hard interlayer). The interface dip angle is $\varphi$. There is an interface crack with its original length of $10 \mathrm{~mm}$ in the end of interface and the direction of crack is similar with the interface. In the calculation, $\varphi$ is given $\pm 30^{\circ}, \pm 45^{\circ}$ and $\pm 60^{\circ}$. When the $\varphi$ is given a positive number, the direction of dip of interface and original crack is upward, namely to the salt rocks. When the $\varphi$ is given negative numbers, the direction of dip of interface and original crack is downward, namely to the interlayer. Quadrilateral plane stain element is used in this model. Pressure equaling 0.2MPa is set on both the top and bottom surfaces of the model. Other materials parameters referred to calculation are showed in Table 1.

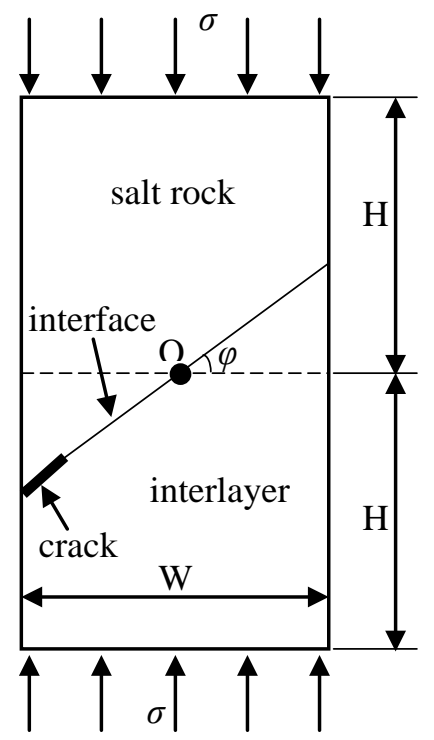

Fig. 1 Schematic diagram of calculation model

Table 1 Material parameters of calculation model

\begin{tabular}{cccccc}
\hline & $\begin{array}{c}\text { Elastic } \\
\text { modulus / } \\
\text { GPa }\end{array}$ & $\begin{array}{c}\text { Poisson' } \\
\text { s ratio }\end{array}$ & $\begin{array}{c}\text { Maximum } \\
\text { allowable principal } \\
\text { stress / Mpa }\end{array}$ & $\begin{array}{c}\text { Critical energy } \\
\text { release rates in } \\
\text { Mode I / N·mm }\end{array}$ & $\begin{array}{c}\text { Critical energy } \\
\text { release rates in } \\
\text { Mode II / N·mm }\end{array}$ \\
\hline $\begin{array}{c}\text { Salt rock } \\
\text { Weak interlayer }\end{array}$ & 16 & 0.35 & 0.09 & 0.05556 & 0.01389 \\
Hard interlayer & 14 & 0.4 & 0.065 & 0.01786 & 0.07143 \\
\hline
\end{tabular}

Assumed that the interlayer is weak interlayer, three kind of interface strengths are taken into consideration in this paper including one which is lower than both the strengths of salt rock and interlayer, one higher than them and one between them (in the following part, they are named as low strength, high strength and medium strength). Detailed strength parameters of interface are shown in Table 2.

Table 2 Strength parameters of interface

\begin{tabular}{ccccccc}
\hline & $\begin{array}{c}\text { Normal } \\
\text { stiffness } \\
\text { / GPa }\end{array}$ & $\begin{array}{c}\text { Tangent } \\
\text { stiffness } \\
\text { / GPa }\end{array}$ & $\begin{array}{c}\text { Critical } \\
\text { normal stress } \\
\text { / Mpa }\end{array}$ & $\begin{array}{c}\text { Critical } \\
\text { tangent stress } \\
\text { / Mpa }\end{array}$ & $\begin{array}{c}\text { Critical normal } \\
\text { fracture energy / } \\
\mathrm{N} \cdot \mathrm{mm}^{-1}\end{array}$ & $\begin{array}{c}\text { Critical tangent } \\
\text { fracture energy / } \\
\mathrm{N} \cdot \mathrm{mm}^{-1}\end{array}$ \\
\hline low strength & 10 & 10 & 0.05 & 0.75 & 0.7 & 0.7 \\
high strength & 70 & 70 & 0.325 & 0.488 & 2.8 & 2.8 \\
medium strength & 15 & 15 & 0.0775 & 0.116 & 1.1 & 1.1 \\
\hline
\end{tabular}




\section{Results and Discussion}

\subsection{Effect of Interlayer Strength.}

First of all, models with high interface strength and $\varphi=45^{\circ}$ are taken as examples to analyze the influence of interlayer strength on crack extension. Crack propagation results in salt rocks with different interlayers are shown in the Figure 2.

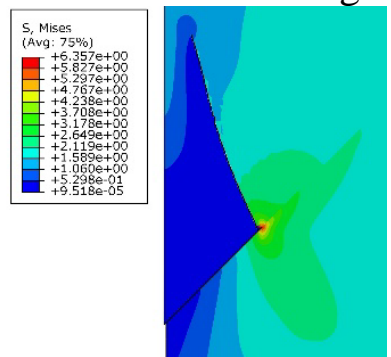

(a) weak interlayer

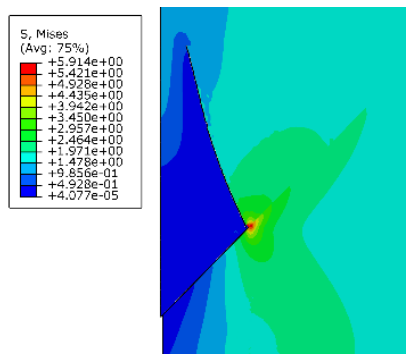

(b)hard interlayer

Fig.2 Crack propagation results in salt rocks with different interlayers

It is indicated from Figure 2 that in the salt rocks containing different interlayers, the crack extension direction and stress fields around cracks are all similar. According to simulation result, the initiation angles of cracks are all $68^{\circ}$ in the two circumstances. The overall crack length in the weak interlayer is $26.2 \mathrm{~mm}$, a little bit longer than $23.6 \mathrm{~mm}$, that in the circumstance of hard interlayer.

\subsection{Effect of Interface Strength.}

Crack propagation results in salt rocks with different interface strengths are shown in Figure 3. And in these models, all the interlayers are weak and $\varphi=45^{\circ}$.It is known from Figure 3 that the length of crack will increase with the growth of interface strength. The crack extension trends are similar under those three interface strengths. According to calculation, the crack initiation angles of low strength model, medium strength model and high strength model are $70^{\circ}, 68^{\circ}$ and $69^{\circ}$. These three crack initiation angles are approximately equal.

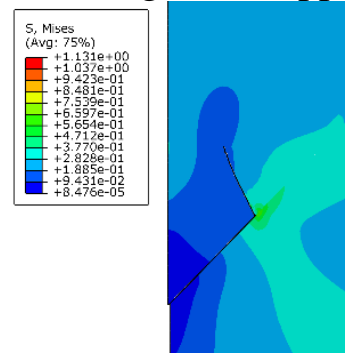

(a) low strength

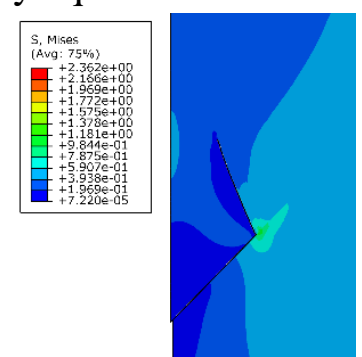

(b) medium strength

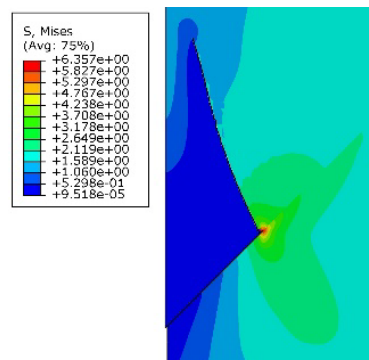

(c) high strength

Fig.3 Crack propagation results in salt rocks with different interface strengths

\subsection{Effect of Interface Dip Angle.}

Crack growth results with different interface dip angles are shown in Figure 4. Weak interlayer and high strength interface are used in these six models. It can be seen from the figure that when the interface tilts upward, the new crack initiates in the upper surface of original crack and extends upward. The new crack initiates in the lower surface of original crack and extends downward when the interface tilts downward. The two propagation paths, upward and downward, are similar when $|\varphi|$ is the same number.

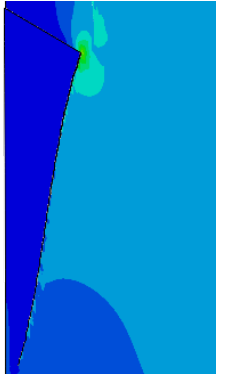

(a) $\varphi=-30^{\circ}$

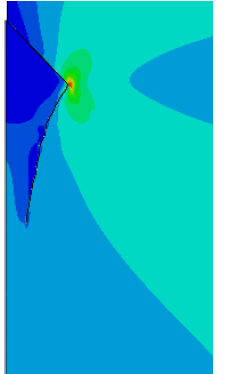

(b) $\varphi=-45^{\circ}$

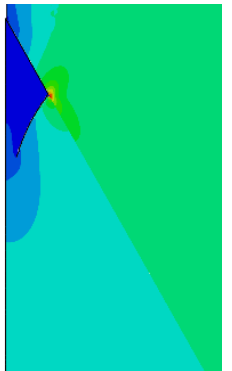

(c) $\varphi=-60^{\circ}$

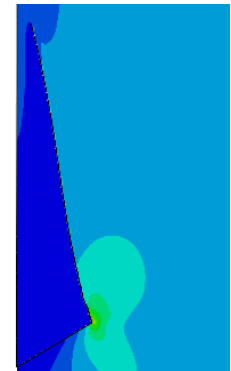

(d) $\varphi=30^{\circ}$

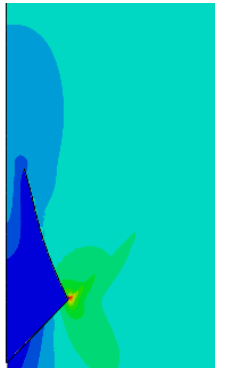

(e) $\varphi=45^{\circ}$

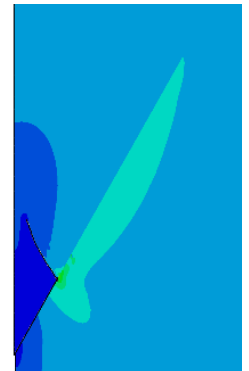

(f) $\varphi=60^{\circ}$

Fig.4 Crack growth results with different interface dip angles 
The overall length and initiation angle of cracks are all showed in Table 3. From the table, it can be inferred that with increase of $|\varphi|$, the length and initiation angle of cracks all decrease. When the $|\varphi|$ is the same number with opposite sign symbols, the initiation angles of cracks are almost the same. However, the crack length will be shorter when the interface tilts downward.

Table 3 Crack length and initiation angles with different interface dip angles

\begin{tabular}{ccc}
\hline$\varphi /^{\circ}$ & Crack initiation angle $^{\circ}$ & Crack length $/ \mathrm{mm}^{\circ}$ \\
\hline-30 & 78 & 44.7 \\
-45 & 71 & 25.4 \\
-60 & 65 & 17.5 \\
30 & 77 & 45.7 \\
45 & 69 & 26.2 \\
60 & 66 & 18.0 \\
\hline
\end{tabular}

\section{Summary}

Using software Abaqus and traction-separation cohesive model within the XFEM framework, interface crack propagation process in layered salt rock is simulated. On the basis of simulation results, it is discussed in this paper about the effects of the three elements---- interlayer strength, interface strength and interface dip angle. Conclusions are as follows:

(1) Under compress and shear load, new cracks appeared deviate from the original one and bend while propagation process. Finally, the new cracks trend to follow the direction of compression stress.

(2) The influence of interlayer strength on crack extension is mainly reflected on the length of cracks. The length of cracks under the weak interlayer circumstance is longer than that under the hard interlayer circumstance.

(3) With the increase of interface strength, the length of cracks will also increase with initiation angles of cracks going similar.

(4) When the interface tilts upward, the cracks extend upward too. The cracks extend downward with the downward tilt of interface. With the increase of inclination of interface, the length and initiation angles of cracks will decrease.

\section{References}

[1]. Thoms R L, Gehle R M. A brief history of salt cavern use. The 8th World Salt Symposium. [S. 1.], 2000, p. 207-214.

[2]. Mingming Tang, Zhiyin Wang, Guosheng Ding. Experimental study of full process of strain of rock salt and salt-mudstone interlayer in Huai' an salt mine. Chinese Journal of Rock Mechanics and Engineering. Vol. 29 (2010) No. 1, p. 2712-2719. (in Chinese)

[3]. Junru Yang, Kangkang Qian, Zhaoqian Li, et al. Status in quo of interface crack propagation of isotropic bi-material. Coal Mine Machinery. Vol. 27 (2006) No. 7, p.54-56. (in Chinese)

[4]. Qing Yang, Chao Wang. Numerical analysis of mixed mode fracture under compression-shear loading based on extended finite element method. Rock and Soil Mechanics. Vol. 32 (2011) No. S2, p. 568-572. (in Chinese)

[5]. Lin Li, Jie Chen, Deyi Jiang, et al. Analysis of surface crack growth in layered salt rock under uniaxial compression. Rock and Soil Mechanics. Vol. 32 (2011) No. 5, p. 1394-1398. (in Chinese)

[6]. Ruidong Peng, Zhide Wu, Hongwei Zhou, et al. Meso-experimental investigation on crack evolution in bedded salt rock. Chinese Journal of Rock Mechanics and Engineering. Vol. 30 (2011) No. S2, p. 3953-3959. (in Chinese) 